\section{Are the serious problems in cancer survival partly rooted in gatekeeper principles?}

As an experienced GP, I disagree with two aspects of this interesting paper.'

First, rather than being an 'unexpected side effect' of gatekeeping, more numerous delayed diagnoses are the inevitable price of fewer unnecessary investigations, that is one of the goals of gatekeeping. We need a study comparing the harm done by investigations (though this could never include patients' unnecessary worry and waste of time) in countries with and without gatekeepers, before deciding which system is superior.

Second, I do not believe that the authors' suggested explanations for delayed investigation and referral, such as financial constraints and fear of being negatively judged by doctors in the secondary sector as referring unnecessarily', are likely to be supported by the future research they wisely recommend. Instead, I think it will show that most of us try to work with individual patients to weigh up the chance of benefit from an investigation against its possible harms. Thus we already act as advisers who counsel the patients on what to do, as the authors recommend, with cost-effectiveness a very secondary consideration.

The public perception of our role as "'keepers" simply rationing care' is already growing more prevalent in anticipation of GP commissioning. I hope this paper will not be cited in support of this unhelpful and illfounded view.

\section{Louisa Polak,}

GP, Nayland Surgery, C06 4LA.

E-mail: lpolakadoctors.org.uk

\section{REFERENCE}

1. Vedsted $P$, Olesen F. Are the serious problems in cancer survival partly rooted in gatekeeper principles? An ecologic study. Br J Gen Pract 2011; DOI: 10.3399/bjgp11X588484.

DOI: 10.3399/bjgp11X606500

Thank you for this important and interesting paper. ${ }^{1}$ Can I ask at what point in the course of the illness and by what criteria cancer was diagnosed? In my practice in Edinburgh, the diagnosis of cancer is based on histological examination of tissue obtained by a specialist. Imagine two patients: one patient in a system without a gatekeeper presents directly to a specialist who takes a biopsy that shows cancer; the patient dies 53 weeks after presentation and is therefore alive at 1 year. The other patient, in my practice, sees me initially and is referred and, 2 weeks later, sees a hospital specialist who takes a biopsy; this second patient dies 53 weeks after presentation to me, that is 51 weeks after seeing the specialist and having cancer diagnosed. Apparently the first patient survives 53 weeks and the second patient 51 weeks. I'd be grateful for a comment on this.

\section{Wilfred Treasure,}

\section{Muirhouse Medical Group, 1 Muirhouse}

Avenue, Edinburgh, EH4 4PL.

E-mail: wtreasureagmail.com

\section{REFERENCE}

1. Vedsted $P$, Olesen F. Are the serious problems in cancer survival partly rooted in gatekeeper

principles? An ecologic study. Br J Gen Pract 2011; DOI: 10.3399/bjgp11X588484.

DOI: 10.3399/bjgp11X606519

The paper by Vedsted and Olesen ${ }^{1}$ raises serious questions about GPs and their gatekeeper role. Clearly, if the gatekeeper function is acting simply as a delaying tactic then it is difficult to justify its presence. If the delay leads to harm, such as delayed diagnosis and increased mortality, then it is an example of a medical system causing harm, and that would need to be reviewed, and maybe removed.

The data that Vedsted and Olesen use are from some time ago, reflecting practice conditions and outcomes in the 1990s. Is no more recent data available to see what is happening currently?

In the UK, GPs now have access to the 2week rule system for urgent referrals and growing access to detailed diagnostic scans and tests. Our means to diagnosis are improving, but we do not yet know if we use them well.

Perhaps the key need now for primary care in the UK, and the world, is to focus its effort more clearly on the diagnostic activity, and its accuracy of problem definition. The current short, crowded primary care consultation in the UK is an obstacle to allowing doctors sufficient thinking time to assess symptoms and their significance, both to the patient and in terms of likely pathology. ${ }^{2,3}$ The problem of delayed diagnoses may not be gatekeeping. but rushing, and thereby failing to define the problem properly. Perversely, we seem to have built a UK medical system based on rushing rather than thinking and in doing so achieved a reduction in both our sensitivity for and specificity of diagnosis. This may appear cheap, but it may actually be costing more to run, as referrals may become a displacement mechanism for time stressed doctors, rather than a carefully formulated question to ask a specialist.

Have we overvalued speed and quantity in medicine thereby actually reducing our quality and effectiveness?

\section{Peter Davies,}

FRCGP, GP, Keighley Road Surgery, Illingworth, Halifax, GP Appraisal Lead, Calderdale PCT, Calderdale Commissioning Consortium, Provost, Yorkshire Faculty, RCGP.

E-mail: md014j1265ablueyonder.co.uk

\section{REFERENCES}

1. Vedsted $P$, Olesen F. Are the serious problems in cancer survival partly rooted in gatekeeper principles? Br J Gen Pract 2011; DOI 10.3399/bjgp11x588484

2. Esmail A. Longer appointments for all. J R Soc Med 2006; 99(12): 644-645.

3. Davies P. The beleaguered consultation. $\mathrm{Br} J \mathrm{Gen}$ Pract 2006; 56(524): 226-229.

DOI: 10.3399/bjgp11X606528

\section{Authors' response}

We are grateful for the debate about positive and negative aspects of gatekeeping raised in three responses to our paper, ${ }^{1}$ and we agree with the important research questions raised in these. First, let us stress that we are strong advocates of the gate-adviser or gatekeeper system, meaning that if any decision-makers will use our paper as an argument for removing the gate-adviser they have simply misunderstood the paper.

We want research that contributes to improving a basically good system. All three 
letters strongly support the need for such research.

Our own primary hypothesis is that healthcare planners have used the easy access to the frontline doctor as an excuse for long waiting lists for complicated clinical trajectories. But we also raise the question whether we as GPs have found the correct balance between necessary investigations and the protection of patients against unnecessary investigations. This is an important, but yet not fully answered, research question that is also raised in the letter from Polak. ${ }^{2}$ We sincerely support the call for more research in the net effect of better access to diagnostic procedures.

Polak also points to the crucial question whether people may have an increasing perception of GPs as being rationing 'keepers' only. That this may have an effect on the way patients seek help is in accordance with new research. ${ }^{3}$ People may think that the GP is some kind of 'barrier' to medical care access and thus postpone attending the GP. Or people experience their nice and friendly GP as very busy and do not want to disturb them, exactly the point also made by Davies. ${ }^{4}$

One of the challenges in comparing differences between countries is the possible difference in how diagnoses are registered. Treasure ${ }^{5}$ has a very important methodological point in asking whether the 1 -year survival is higher in the nongatekeeper systems simply due to lead time bias. Lead time bias is definitely a possible explanation for the outcome of these types of comparative studies. The question is, however, whether such lead time bias can explain differences between countries of $5-10 \%$ in relative survival. A recent simulation study ${ }^{6}$ found that the difference in registration of a breast cancer diagnosis should be unlikely large if it should explain the differences between UK and Sweden. However, the effect of lead time bias in comparisons needs much more rigorous research.

We strongly agree with Davies that our data should be replicated using newer data and also data on other serious diseases. New research should also address if different remuneration systems may have impact on the quality of gatekeeping.

We were happy to see three academically well argued responses to our paper. A fourth response by Manassiev lposted on the BJGP Discussion Forum) seems to be very little in favour of discussing whether there could be side effects of gatekeeping. In many ways the response speaks for itself. In a proper way we point out that our study is an ecologic study.
The use of quintiles in the paper by Møller et al does not change anything as we used this in our calculations. We do not think that use of the 1- and 5-year relative survival of lip cancer would improve the paper as suggested by Manassiev. Manassiev may have different memories about gatekeeping in some countries, but we prefer research published in the literature. We have written our arguments for the use of 1-year survival and we kindly ask our readers to check them and compare with Manassiev's not quite academic approach. It is not correct that the majority of sufferers of the top four cancers (lung, breast, prostate, and colon) would survive 1 year probably whatever the health system'. We do not agree with Manassiev about the incompetence of editors and reviewers and we trust that many readers are able to read papers without having passed Manassiev's research school on the shape of the earth.

In conclusion, we must realise that general practice has several key roles. One important role is to be aware of new, rare, but serious diseases that, in a timely way, should be guided through the healthcare system without delay that may influence prognosis. We need much more research on the impact of different organisational models on this key role. $^{8}$

\section{Peter Vedsted,}

Aarhus University, Research Unit for General Practice, Bartholins Allé 2, Aarhus, 8000, Denmark.

E-mail: p.vedstedlaalm.au.dk

Frede Olesen,

University of Aarhus, Research Unit for General Practice, Aarhus C, 8000, Denmark.

\section{REFERENCES}

1. Vedsted $P$, Olesen F. Are the serious problems in cancer survival partly rooted in gatekeeper principles? An ecologic study. Br J Gen Pract 2011; DOI: 10.3399/bjgp11X588484.

2. Polak L. Are the serious problems in cancer survival partly rooted in gatekeeper principles? [Letter] $\mathrm{Br} J$ Gen Pract 2011; 61(592): 661.

3. Andersen RS, Vedsted P, Olesen F, et al. Does the organizational structure of health care systems influence care-seeking decisions? A qualitative analysis of Danish cancer patients' reflections on care-seeking. Scand J Prim Health Care 2011; 29(3): 144-149

4. Davies P. Are the serious problems in cancer survival partly rooted in gatekeeper principles? [Letter] $\mathrm{Br} \mathrm{J}$ Gen Pract 2011; 61(592): 661.

5. Treasure W. Are the serious problems in cancer survival partly rooted in gatekeeper principles? [Letter] Br J Gen Pract 2011; 61(592): 661.
6. Woods LM, Coleman MP, Lawrence G, et al. Evidence against the proposition that 'UK cancer survival statistics are misleading': simulation study with National Cancer Registry data. BMJ 2011; 342: d3399.

7. Møller $\mathrm{H}$, Linklater KM, Robinson D. A visual summary of the EUROCARE-4 results: a UK perspective. Br J Cancer 2009; 101 (Suppl 2): S110-114.

8. Olesen F. Putting research into primary care practice. BMJ 2011; 343: d3922

DOI: 10.3399/bjgp11X606537

\section{Extent of cotton-bud use in ears}

The use of cotton buds inside ears has widely been condemned worldwide by otolaryngologists. This is due to well documented complications including trauma, impacted ear wax, infection, and retention of the cotton bud. ${ }^{1}$ The most common mode of accidental penetrating ear injury in children is cotton-bud induced. ${ }^{2}$ Trends in cotton-bud usage have been studied previously, but have only focused on ear, nose, and throat (ENT) patients. ${ }^{3}$ In recent times, manufacturers have heeded the advice of the otolaryngologist and have consequently relayed warnings to the public. We conducted a survey to investigate the extent of 'Q-tip' cotton bud public use in ears and the awareness of associated complications.

Between January and August 2009, confidential questionnaires were given to patients at three primary care centres in the south east of England. The response rate was $80 \%$ (239/300). Ages ranged from 17 to 87 years, with a mean of 41.1 years. There were 144 (60\%) female and 95 (40\%) male responders. An alarming $68 \%$ admitted using cotton buds in their ears, with $76 \%$ of users using them at least weekly, if not more frequently. The primary reason (96\%) given for using cotton buds was to remove earwax.

It is evident that there is a public perception that the ear requires regular cleaning. However, our knowledge dictates that earwax is produced in the outer part of the canal and migrates out with the epithelium towards the pinna. Other reasons included relieving an itch and drying the ear. Our survey showed that cotton-bud users were aware of $52 \%$ of the potential complications, whereas non cotton-bud users were aware of $59 \%$ of potential complications. There was no significant association between awareness of complications and cotton-bud use $l \chi^{2}=2.23$, $\mathrm{df}=3, P=0.53)$.

Despite manufacturers' warnings, use of 